ARTICLE

Received 6 Apr 2016 | Accepted 31 Aug 2016 | Published 20 Oct $2016 \quad$ DOl: 10.1038/ncomms13065 OPEN

\title{
A sulfur host based on titanium monoxide@carbon hollow spheres for advanced lithium-sulfur batteries
}

Zhen Li ${ }^{1}$, Jintao Zhang ${ }^{1}$, Buyuan Guan¹, Da Wang ${ }^{2}$, Li-Min Liu ${ }^{2} \&$ Xiong Wen (David) Lou ${ }^{1,3}$

Lithium-sulfur batteries show advantages for next-generation electrical energy storage due to their high energy density and cost effectiveness. Enhancing the conductivity of the sulfur cathode and moderating the dissolution of lithium polysulfides are two key factors for the success of lithium-sulfur batteries. Here we report a sulfur host that overcomes both obstacles at once. With inherent metallic conductivity and strong adsorption capability for lithium-polysulfides, titanium monoxide@carbon hollow nanospheres can not only generate sufficient electrical contact to the insulating sulfur for high capacity, but also effectively confine lithium-polysulfides for prolonged cycle life. Additionally, the designed composite cathode further maximizes the lithium-polysulfide restriction capability by using the polar shells to prevent their outward diffusion, which avoids the need for chemically bonding all lithium-polysulfides on the surfaces of polar particles.

\footnotetext{
${ }^{1}$ School of Chemical and Biomedical Engineering, Nanyang Technological University, 62 Nanyang Drive, Singapore 637459, Singapore. ${ }^{2}$ Beijing Computational Science Research Center, Beijing 100084, China. ${ }^{3}$ State Key Laboratory of Silicon Materials, School of Materials Science and Engineering, Zhejiang University, Hangzhou, 320027, China. Correspondence and requests for materials should be addressed to X.W.L. (email: xwlou@ntu.edu.sg) or to L.-M.L. (email: limin.liu@csrc.ac.cn, for correspondence regarding DFT calculations).
} 
$\mathrm{R}$ echargeable battery systems are a vital part of many emerging applications, such as grid electrical storage and electric vehicles. Among existing electrochemical systems, lithium-sulfur (Li-S) batteries show advantages for nextgeneration electrical energy storage and conversion due to their high theoretical energy density, low cost and environmental friendliness ${ }^{1}$. However, the commercialization of the rechargeable Li-S battery is still hindered by three main issues of: (a) the inherent poor electronic conductivity of sulfur and its end products of discharge $\left(\mathrm{Li}_{2} \mathrm{~S} / \mathrm{Li}_{2} \mathrm{~S}_{2}\right)$, (b) the dissolution of intermediate lithium polysulfides (LiPSs), and (c) large volumetric expansion of $\sim 80 \%$ upon full lithiation. These issues bring about serious self-discharge, low Coulombic efficiency and rapid decline of capacity upon cycling ${ }^{2}$. Strenuous efforts have been devoted to improve the electrochemical performance of $\mathrm{Li}-\mathrm{S}$ batteries, including composing sulfur with conductive materials ${ }^{3-7}$, constructing LiPSs blocking interlayers ${ }^{8-11}$, developing new electrolytes $^{12-14}$ and applying functional binders ${ }^{15-17}$. Among these methods, the most popular strategy is composing sulfur with carbonaceous materials $6,7,18,19$, since their intrinsic good conductivity and diversity in nanostructures make the carbon materials very attractive $e^{19-22}$. However, the carbon/sulfur composite cathodes still generally suffer from rapid capacity fading over long-term cycling, because the nonpolar carbon can only provide weak physical adsorption to the polar LiPSs ${ }^{23}$. Once LiPSs are solvated, they can easily dissolve into the organic electrolyte from the electrode surface and diffuse away. Subsequent reutilization of LiPSs for capacity contribution will become very difficult due to the repulsion between the polar reactants and the nonpolar conductive surface ${ }^{24}$.

Recently, it has been realized that polar functional groups/ surfaces can significantly increase the chemical interaction between polysulfides and the substrates ${ }^{23,24}$, and many efforts have been expended to develop sulfur hosts with strong chemical adsorption capability for LiPSs. For instance, heteroatom doping ${ }^{25-27}$ and surfaces functionalization ${ }^{28-30}$ of carbon materials lead to significant improvement of chemical adsorption of LiPSs. Taking advantage of the Lewis acid-base interactions with polysulfides, metal organic frameworks ${ }^{31}$, MXene nanosheets ${ }^{32}$ and metal hydroxides ${ }^{33,34}$ have been employed as sulfur hosts and achieved good cycling stability. Polar metal oxides/sulfides, such as $\mathrm{SiO}_{2}$ (ref. 35), $\mathrm{TiO}_{2}$ (ref. 36), indium tin oxide ${ }^{37}, \mathrm{MnO}_{2}$ (refs 38,39), $\mathrm{TiS}_{2}$ (ref. 40) and $\mathrm{CoS}_{2}$ (ref. 41) can also adsorb polysulfides more tightly than carbon substrate, and provide significantly improved cycling properties. However, many metal oxides/sulfides usually have intrinsically poor electrical conductivity, thus the chemically adsorbed polysulfides are difficult to be reduced directly on the hosts' surfaces, resulting relatively lower sulfur utilization. To enhance the electrical conductivity of polar metal oxides/ sulfides for improving the redox kinetics of the electrode, $\mathrm{Ti}_{4} \mathrm{O}_{7}$ nanoparticles ${ }^{42,43}$ and $\mathrm{Co}_{9} \mathrm{~S}_{8}$ nanosheets ${ }^{44}$ have been employed as new concept sulfur hosts with both good conductivity and polar nature in one host. So far, utilization of polar materials with LiPSs anchoring abilities is viewed as an important strategy to confine polysulfide species and avoid their dissolution ${ }^{24}$. Yet the study of polar materials as sulfur hosts is still in its early stages. Most polar materials are applied just in the irregular particulate form, which can only adsorb LiPSs near the surfaces (Fig. 1a). This imposes another important issue one cannot avoid. Specifically, when the sulfur content is higher than a certain value, the polar material would not be able to provide sufficient interfaces to fix all of the LiPSs in the electrode (Fig. 1b). Therefore, more efficient LiPSs-trapping structures are in urgent need to solve the technical challenges of sulfur cathodes in Li-S batteries.

One practical way is to apply the least amount of polar materials as the shell of nanochambers (Fig. 1c). The polar hollow nanostructure has at least two main advantages: (i) the polar shell only needs to adsorb part of the LiPSs near the entrance, and then the internal deep-seated LiPSs will be naturally hindered from dissolution, (ii) the large void space of hollow materials not only allows loading of relatively higher content of sulfur, but also accommodates the large volumetric expansion of sulfur during lithiation. Beyond that, to obtain higher utilization of sulfur for higher capacities, the electrical conductivity of the polar hollow host also needs to be enhanced (Fig. 1d). Unfortunately, it is very challenging to synthesize metal oxides with both hollow nanostructure and high conductivity, due to the required high temperature annealing process for producing conductive metal oxides like Magnéli phase titanium oxides $\left(\mathrm{Ti}_{n} \mathrm{O}_{2 n-1}, n=4 \sim 10\right)$ (ref. 45). Therefore, the application of hollow nanostructured conductive metal oxides as the sulfur host is rarely reported.

Herein, we design and synthesize polar hollow nanospheres with highly conductive shells constituted of titanium monoxide (TiO) and carbon as the sulfur host. With a high density of oxygen and titanium vacancies, the rock-salt structured stoichiometric $\mathrm{TiO}$ exhibits excellent electrical conductivity, which is nearly one order of magnitude higher than that of
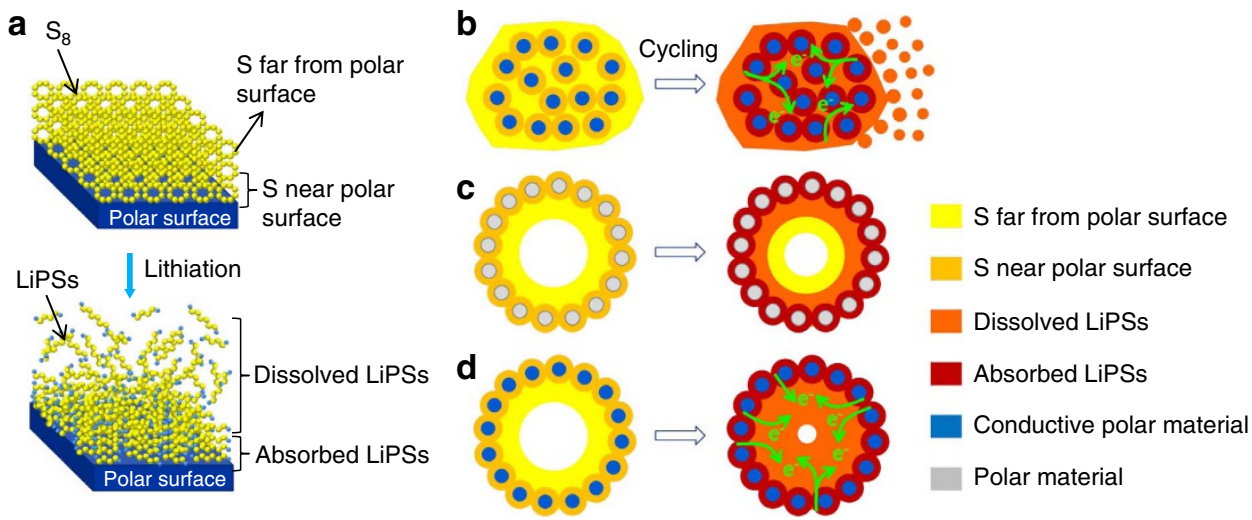

Figure 1 | Schematic illustration of the adsorption limitation of LiPSs for polar hosts. (a) LiPSs can be chemically adsorbed only when they are close enough to the polar surface, LiPSs far from the polar surface cannot be effectively anchored during the cycling. (b) Conductive polar nanoparticles can chemically adsorb LiPSs near their surfaces. When the sulfur content of the composite exceeds the limit, the extra LiPSs would dissolve into the organic electrolyte. (c) The hollow polar structure can bond with LiPSs near the surface, and effectively restrict the diffusion of the inner LiPSs. However, the low conductivity of the host hinders high sulfur utilization. (d) The conductive polar hollow structure inherits both advantages of b,c. 
Magnéli phase $\mathrm{Ti}_{n} \mathrm{O}_{2 n-1} \quad(n=4 \sim 10) \quad$ (refs 45,46), and its chemical resistance is similar to that of $\mathrm{Ti}_{4} \mathrm{O}_{7}$ (ref. 47). Density functional theory (DFT) calculations indicate that $\mathrm{TiO}$ can provide more effective adsorption of LiPSs than $\mathrm{TiO}_{2}$. Additionally, unlike previously reported granular sulfur hosts $^{42,43}$, the designed titanium monoxide@carbon hollow spheres (TiO@C-HS) maximizes the LiPSs restriction capability by using just the polar shells to effectively prevent the outward diffusion of LiPSs, which breaks the limitation of chemically bonding all LiPSs on the surfaces of polar particles. As a result, the TiO@C-HS/S composite cathode delivers excellent comprehensive electrochemical properties even without using any other additive material.

\section{Results}

Materials synthesis and characterization. The synthesis strategy of the TiO@-HS/S composite is illustrated in Fig. 2a. Uniform polystyrene (PS) spheres are prepared as the template via a modified method reported elsewhere ${ }^{48}$. As shown in the scanning electron microscopy (SEM) and transmission electron microscope (TEM) images in Fig. 2b-e, the PS nanospheres are highly uniform with an average diameter of about $490 \mathrm{~nm}$. Subsequently, a thin layer of amorphous $\mathrm{TiO}_{2}$ is uniformly coated on the surface of PS nanospheres through a cooperative assembly-directed strategy ${ }^{49}$. The PS@ $\mathrm{TiO}_{2}$ core-shell structured nanospheres are also nearly monodisperse with an average diameter of about $550 \mathrm{~nm}$ (Fig. $2 \mathrm{f}-\mathrm{h}$ ), indicating that the thickness of the $\mathrm{TiO}_{2}$ shell is around $30 \mathrm{~nm}$, in agreement with the TEM observation (Fig. 2i). After coated with a layer of polydopamine (PDA), the $\mathrm{PS} \mathrm{TiO}_{2} @ \mathrm{PDA}$ sample still well maintains the spherical morphology with smooth surfaces (Fig. 2j-1), indicating PDA is uniformly polymerized outside the PS@TiO ${ }_{2}$ spheres. Since the thickness of the shell on PS is increased to $\sim 40 \mathrm{~nm}$ (Fig. $2 \mathrm{~m}$ ), the thickness of PDA layer is presumed to be about $10 \mathrm{~nm}$.

In order to transform $\mathrm{TiO}_{2}$ to conductive $\mathrm{TiO}$, the PS@TiO $\mathrm{TPDA}_{2}$ sample is annealed in a reductive atmosphere of $\mathrm{N}_{2} / \mathrm{H}_{2}$ (95:5) mixture gas at $1,000{ }^{\circ} \mathrm{C}$ for $4 \mathrm{~h}$. TiO@C-HSs maintain uniform morphology with intact spherical shells, and the average diameter is $\sim 550 \mathrm{~nm}$ (Fig. 3a,b). The hollow structure of TiO@C-HSs can be identified from a broken one (Fig. 3c). The outer PDA layer is transformed into amorphous carbon after the annealing treatment (Fig. 3d). X-ray diffraction analysis (Fig. 3e) indicates that TiO (JCPDS No. 77-2170) is the primary crystalline phase of the product. TEM images show that the obtained TiO@C-HSs has uniform hollow architecture (Fig. 3f), and the inner shell is composed of abundant small nanocrystals (Fig. 3g). The clear lattice fringes with an inter-planar spacing of $0.24 \mathrm{~nm}$ can be readily assigned to the (111) planes of rock-salt TiO (Fig. 3h). It is interesting to note

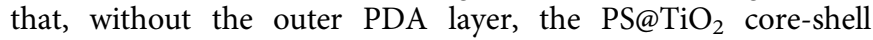
particles will be transformed into large-sized irregular particles after the same high-temperature annealing treatment, and the

a

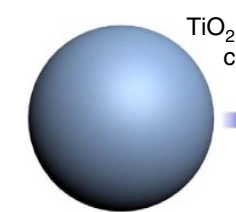

PS
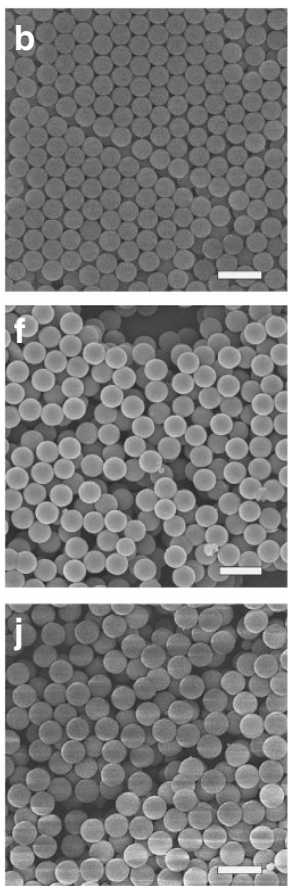

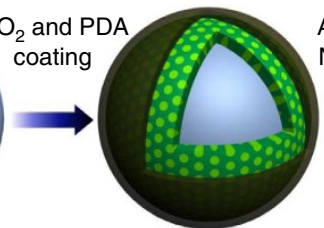

$\mathrm{PS} @ \mathrm{TiO}_{2} @ \mathrm{PDA}$
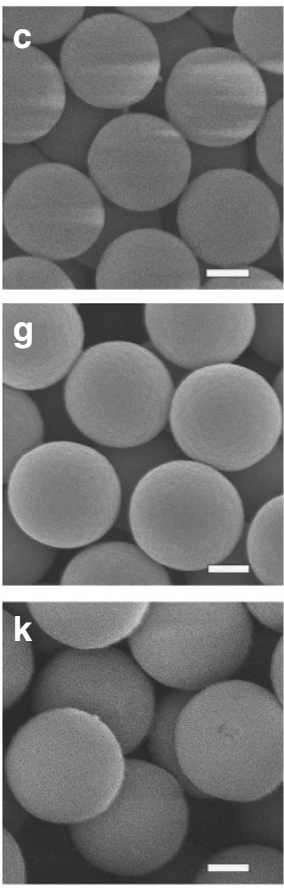

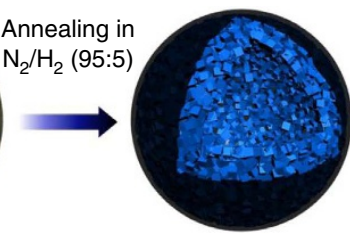

TiO@C-HS
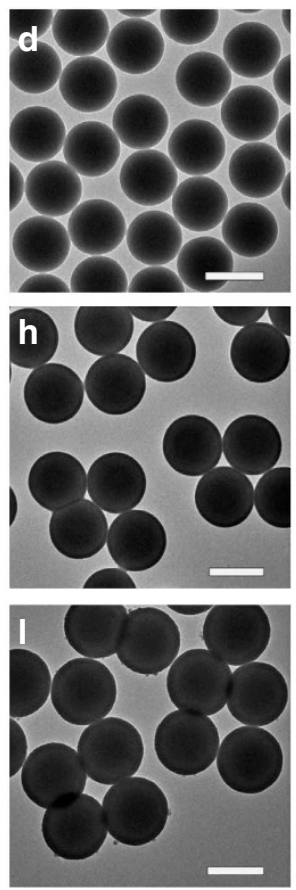

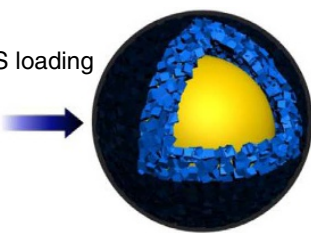

$\mathrm{TiO} @ \mathrm{C}-\mathrm{HS} / \mathrm{S}$
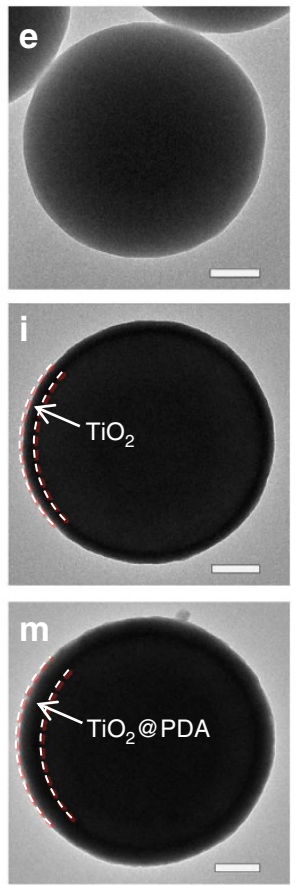

Figure 2 | Synthesis process of the TiO@C-HS/S composite. (a) Schematic illustration of the synthesis process of the TiO@C-HS/S composite. SEM and TEM images of (b-e) PS spheres, (f-i) PS@TiO 2 core-shell spheres and (j-m) PS@TiO $@$ @PDA spheres. Scale bars, $1 \mu \mathrm{m}$ (b,f,j), scale bars, 200 nm $(\mathbf{c}, \mathbf{g}, \mathbf{k})$, scale bars, $500 \mathrm{~nm}(\mathbf{d}, \mathbf{h}, \mathbf{l})$, scale bars, $100 \mathrm{~nm}(\mathbf{e}, \mathbf{i}, \mathbf{m})$. 
a
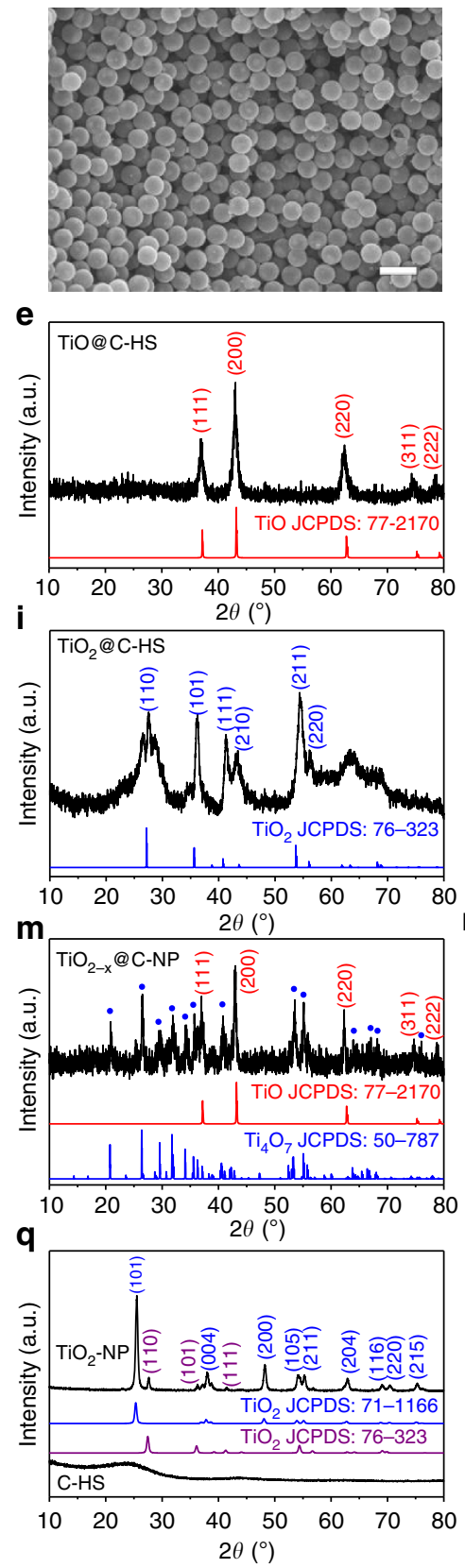

b
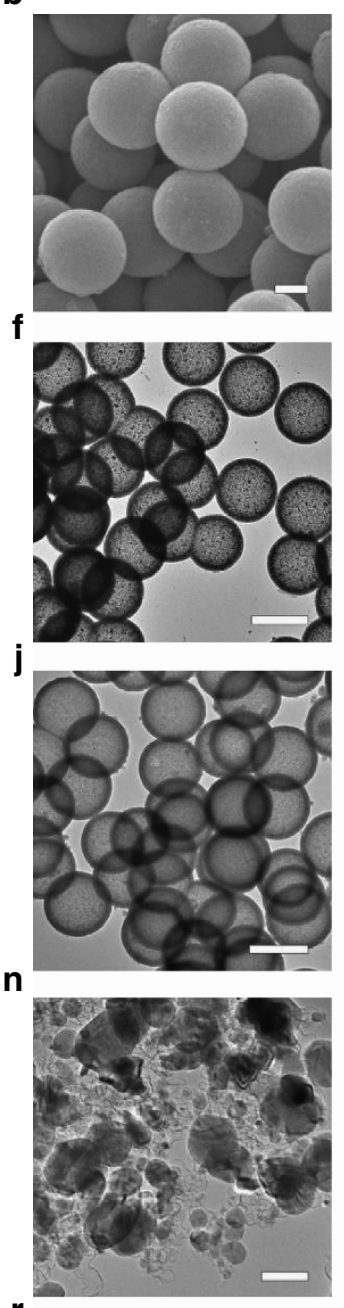

$r$

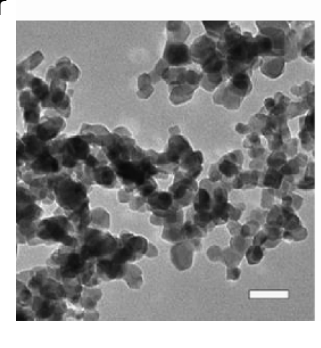

C
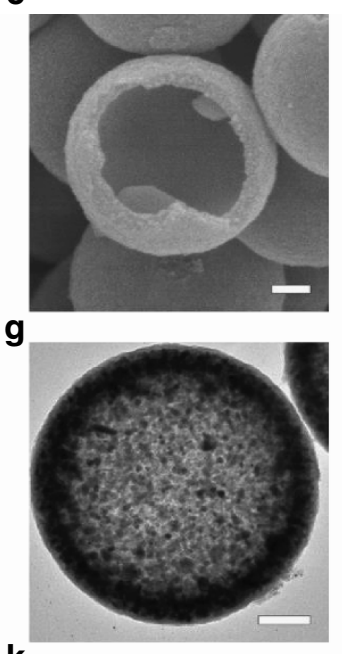

k

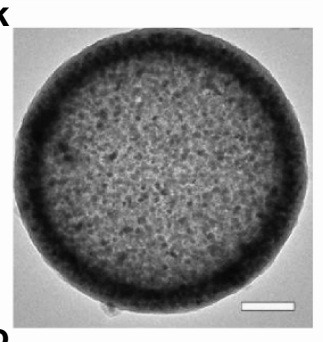

0

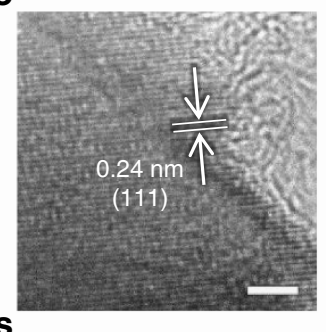

s

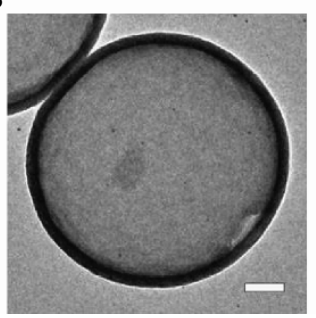

d

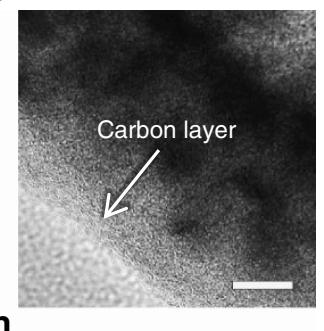

h

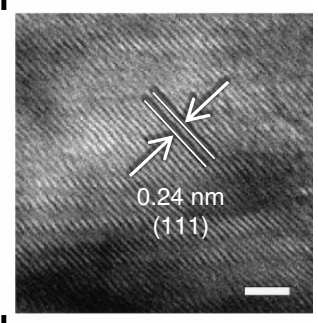

I

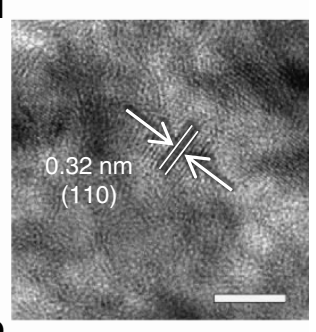

p

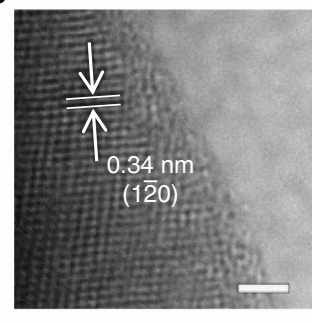

t

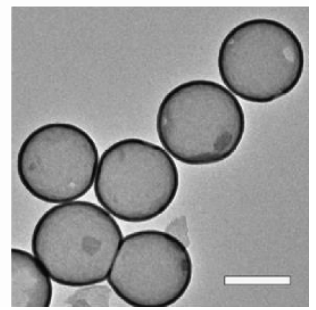

Figure 3 | Characterization of TiO@C-HS and other control host materials. (a-c) SEM images, (e,i,m,q)X-ray diffraction patterns, (d,f-h,j-l,n-p,r-t) TEM images of (a-h)TiO@C-HS, (i-I) TiO $2 @ C-H S,(\mathbf{m}-\mathbf{p}) \mathrm{TiO}_{2-x} @ \mathrm{C}-\mathrm{NP},(\mathbf{q}, \mathbf{r}) \mathrm{TiO}_{2}-\mathrm{NP}$ and (s,t) C-HS. Scale bars, $1 \mu \mathrm{m}(\mathbf{a})$, scale bars, $200 \mathrm{~nm}(\mathbf{b}), \mathrm{scale}$ bars, $100 \mathrm{~nm}(\mathbf{c}, \mathbf{g}, \mathbf{k}, \mathbf{n}, \mathbf{s})$, scale bars, $500 \mathrm{~nm}(\mathbf{f}, \mathbf{j}, \mathbf{t})$, scale bars, $10 \mathrm{~nm}(\mathbf{d})$, scale bars, $5 \mathrm{~nm}(\mathbf{I})$, scale bars, $2 \mathrm{~nm}(\mathbf{h}, \mathbf{o}, \mathbf{p})$, scale bars, $50 \mathrm{~nm}(\mathbf{r})$. a.u., arbitrary unit.

spherical morphology is completely destroyed (Supplementary Fig. 1). Therefore, the presence of the protective outer carbon layer is very crucial for controlling the crystal phase and size of the inner TiO. Thermogravimetric analysis reveals that the carbon content in the TiO@C-HS structure is $\sim 43 \mathrm{wt} \%$ (Supplementary Fig. 2).

To show the structural advantages of TiO@C-HS as the sulfur host, four control samples are also prepared. First, $\mathrm{TiO}_{2} @ \mathrm{C}-\mathrm{HS}$ with a similar hollow structure is prepared by annealing PS@TiO ${ }_{2} @ P D A$ at $900{ }^{\circ} \mathrm{C}$ in the same atmosphere. The crystal phase of the product is rutile $\mathrm{TiO}_{2}$ (Fig. 3i; JCPDS No. 76-323). $\mathrm{TiO}_{2} @ \mathrm{C}-\mathrm{HS}$ also maintains uniform hollow spherical structure (Fig. 3j), while the grain sizes of the $\mathrm{TiO}_{2}$ nanocrystals are smaller than that of TiO@C-HS due to the lower annealing temperature
(Fig. 3k). The lattice fringes of the nanocrystals are correlated to the (110) planes of rutile $\mathrm{TiO}_{2}$ (Fig. 3l). The carbon content of $\mathrm{TiO}_{2} @ \mathrm{C}-\mathrm{HS}$ is $\sim 40 \mathrm{wt} \%$ (Supplementary Fig. 3), which is lower than that of $\mathrm{TiO} @ \mathrm{C}-\mathrm{HS}$ due to the relatively higher weight ratio of oxygen in the $\mathrm{TiO}_{2}$ shell. The second control sample is the carbon coated conductive $\mathrm{TiO}_{2-x}$ nanoparticles $\left(\mathrm{TiO}_{2-x} @ \mathrm{C}-\mathrm{NP}\right)$ synthesized by annealing the commercial $\mathrm{TiO}_{2}$ nanoparticles (Supplementary Fig. 4a,b) with PDA at $1,000^{\circ} \mathrm{C}$ for $4 \mathrm{~h}$ in the same atmosphere of $\mathrm{N}_{2} / \mathrm{H}_{2}$ (95:5). In this sample, a mixture of $\mathrm{TiO}$ and $\mathrm{Ti}_{4} \mathrm{O}_{7}$ is obtained (Fig. $3 \mathrm{~m}$ ), and the carbon content is estimated to be $15-20 \mathrm{wt} \%$ (Supplementary Fig. 3). TEM and SEM images (Fig. 3n, Supplementary Fig. 4c,d) show abundant irregular nanoparticles with diameters from 40 to $150 \mathrm{~nm}$, suggesting an obvious morphological evolution during 
the high temperature reduction treatment. The re-shaped $\mathrm{TiO}_{2-x}$ nanoparticles gain abundant exposed surface without being covered by carbon layers (Supplementary Fig. 5). High resolution TEM (HRTEM) observations further suggest the crystal phases of $\mathrm{TiO}$ (Fig. 3o) and $\mathrm{Ti}_{4} \mathrm{O}_{7}$ (Fig. 3p) nanoparticles in the $\mathrm{TiO}_{2}$ @@C-NP sample. Similar with the $\mathrm{PS} @ \mathrm{TiO}_{2}$ precursor, annealing of bare $\mathrm{TiO}_{2}$ nanoparticles yields very large particle sizes from $200 \mathrm{~nm}$ to $1 \mu \mathrm{m}$ (Supplementary Fig. 4e,f), which once again proves that the carbon layer has an important role on controlling the particle size of titanium oxides during the high temperature treatment. To further demonstrate the effectiveness of the hollow structure and the important role of the polar $\mathrm{TiO}$ layer, commercial $\mathrm{TiO}_{2}$ nanoparticles $\left(\mathrm{TiO}_{2}-\mathrm{NP}\right)$ and pure carbon hollow spheres (C-HS) are applied as another two control groups. The $\mathrm{TiO}_{2}-\mathrm{NP}$ sample has mixed crystal phases of anatase and rutile (Fig. 3q) with particle sizes of $20-30 \mathrm{~nm}$ (Fig. 3r). The C-HS are synthesized by coating PS with PDA, followed by annealing at $900{ }^{\circ} \mathrm{C}$ in $\mathrm{N}_{2}$. The obtained C-HS exhibit an amorphous carbon phase (Fig. 3q) and well maintained hollow structure (Fig. 3s,t).

Sulfur is loaded within the TiO@C-HS host by a modified vapour phase infusion method ${ }^{50}$. SEM observations reveal that the TiO@C-HS/S composite well maintains the original spherical shape with smooth surfaces (Fig. 4a,b), indicating that no extra sulfur exists outside the TiO@C-HS structure. Energy-dispersive X-ray (EDX) spectrum (Fig. 4c) and X-ray diffraction pattern (Fig. 4d) of the TiO@C-HS/S composite prove the presence of sulfur and TiO. TEM images (Fig. 4e,f) show that the contrast of the inner space of TiO@C-HS becomes much darker after sulfur loading, and the crystal nanoparticles of $\mathrm{TiO}$ on the shells cannot be easily identified. The linear scan analysis shows that the distribution of $\mathrm{S}$ is contrary to Ti and C (Fig. 4g,h), indicating that a high content of sulfur is present inside the TiO@C-HS host. The observations demonstrate that sulfur has been successfully accommodated and immobilized within the porous shells and the inner void spaces of TiO@C-HSs. EDX elemental mapping (Fig. 4i) of many TiO@C-HS/S nanospheres shows that sulfur is homogeneously distributed in the TiO@C-HS host. The $\mathrm{TiO}_{2} @ \mathrm{C}-\mathrm{HS} / \mathrm{S}$ composite shows similar morphology with the TiO@C-HS/S (Fig. 4j), again suggesting the excellent sulfur loading capacity of hollow structures. However, for the $\mathrm{TiO}_{2-x} @ \mathrm{C}-\mathrm{NP} / \mathrm{S}$ composite (Fig. 4k) and the $\mathrm{TiO}_{2}-\mathrm{NP} / \mathrm{S}$ composite (Fig. 4l), thick layers of sulfur are evident on the surfaces of the host particles. After composing with sulfur by melt-diffusion at $155^{\circ} \mathrm{C}$, the $\mathrm{C}-\mathrm{HS} / \mathrm{S}$ composite still retains the a
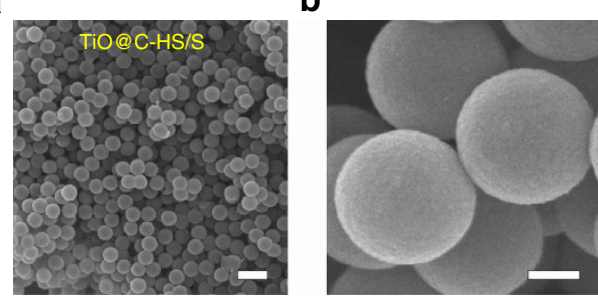

C

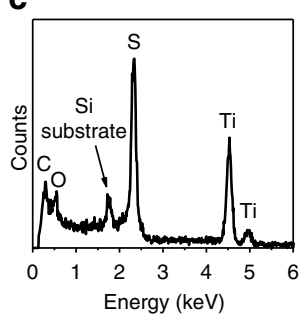

g

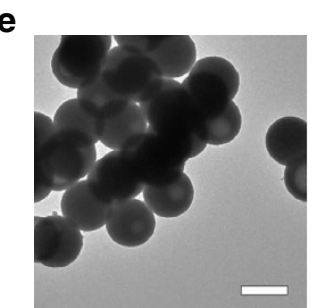

f

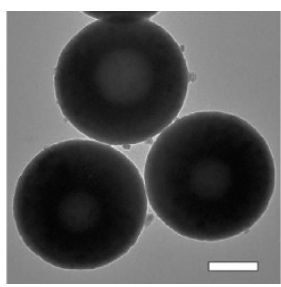

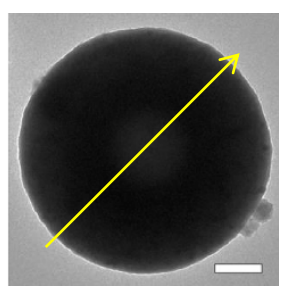

d

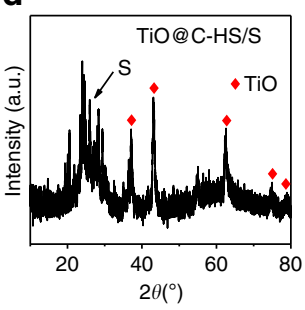

h

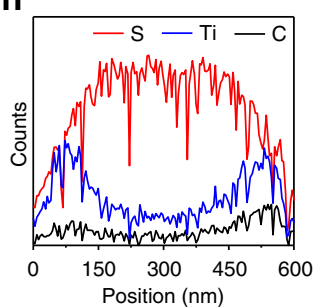

i

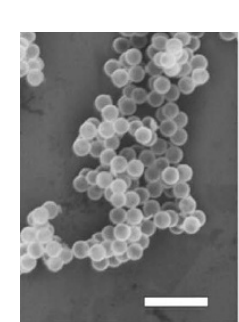

j

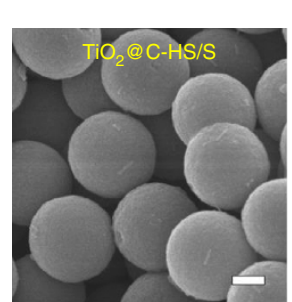

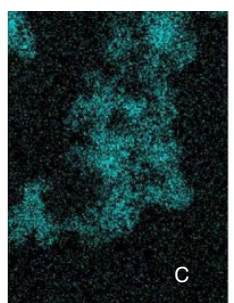

k

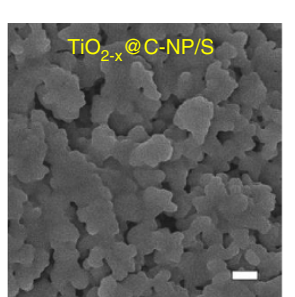

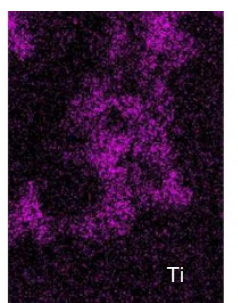

I

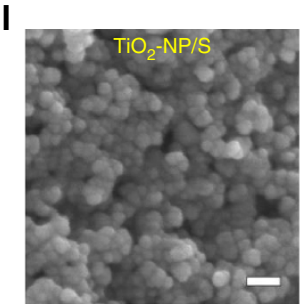

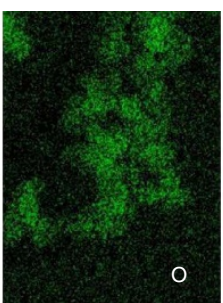

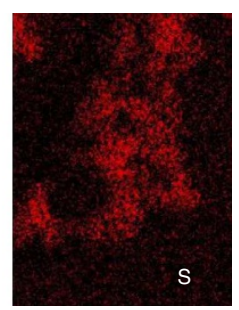

m

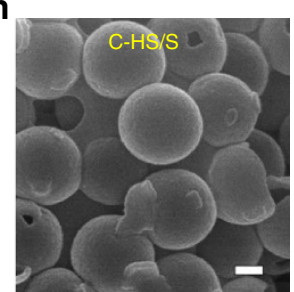

Figure 4 | Characterization of the sulfur-based composite materials. (a,b) SEM image, (c) EDX spectrum, (d) X-ray diffraction pattern, (e-g) TEM images, (h) linear distributions of $\mathrm{S}, \mathrm{Ti}$ and $\mathrm{C}$ along the arrow line on $\mathbf{g}$, and (i) EDX mapping area and corresponding elemental distributions of $\mathrm{C}$, Ti, $\mathrm{O}$ and $\mathrm{S}$ of TiO@C-HS/S. SEM images of (j) TiO $@ @ C-H S / S,(\mathbf{k}) T_{2} \mathrm{TiO}_{2-x} @ \mathrm{C}-\mathrm{NP} / \mathrm{S},(\mathbf{I}) \mathrm{TiO}_{2}-\mathrm{NP} / \mathrm{S}$ and $(\mathbf{m}) \mathrm{C}-\mathrm{HS} / \mathrm{S}$. Scale bars, $1 \mu \mathrm{m}$ (a), scale bars, $200 \mathrm{~nm}$ $(\mathbf{b}, \mathbf{f}, \mathbf{j}, \mathbf{k}, \mathbf{m})$, scale bars, $500 \mathrm{~nm}(\mathbf{e})$, scale bars, $100 \mathrm{~nm}(\mathbf{g}, \mathbf{l})$, scale bars, $2 \mu \mathrm{m}(\mathbf{i})$. 
original spherical morphology (Fig. 4m). To make a fair comparison, the sulfur contents in all five samples are controlled to be $\sim 70 \mathrm{wt} \%$ (Supplementary Fig. 6).

Electrochemical performance. Both the sulfur mass loading and the amount of electrolyte injected in the coin cells are kept the same for all five samples in order to compare and evaluate the structural effects of host materials on the electrochemical properties. From the Nyquist plots (Fig. 5a), it can be observed that the TiO@C-HS/S electrode has the smallest semicircle in high-frequency region, indicating the lowest charge transfer resistance compared with $\mathrm{TiO}_{2} @ \mathrm{C}-\mathrm{HS} / \mathrm{S}$ and $\mathrm{TiO}_{2-} @ \mathrm{C}-\mathrm{NP} / \mathrm{S}^{43}$. Since all cathodes contain approximately the same content of sulfur, the different charge transfer resistances could be attributed to the conductivity of the host materials. Benefitting from the metallic nature of $\mathrm{TiO}$ and the good confinement of sulfur, TiO@C-HS/S shows better ability to facilitate the charge transfer for surface reactions than the other sulfur hosts. On the contrary, due to the relatively poor conductivity of $\mathrm{TiO}_{2}$, the $\mathrm{TiO}_{2}-\mathrm{NP} / \mathrm{S}$ electrode shows the highest charge transfer resistance. Figure $5 \mathrm{~b}$ shows the second-cycle charge/discharge voltage profiles of the TiO@C-HS/S, TiO ${ }_{2} @ \mathrm{C}-\mathrm{HS} / \mathrm{S}, \mathrm{TiO}_{2-x} @ \mathrm{C}-\mathrm{NP} / \mathrm{S}, \mathrm{C}-\mathrm{HS} / \mathrm{S}$ and $\mathrm{TiO}_{2}-\mathrm{NP} / \mathrm{S}$ electrodes at $0.1 \mathrm{C}\left(1 \mathrm{C}=1,675 \mathrm{mAg}^{-1}\right)$. Among all

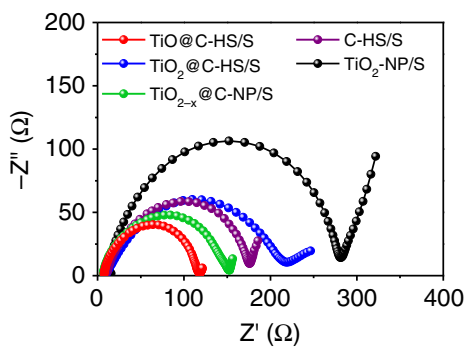

d

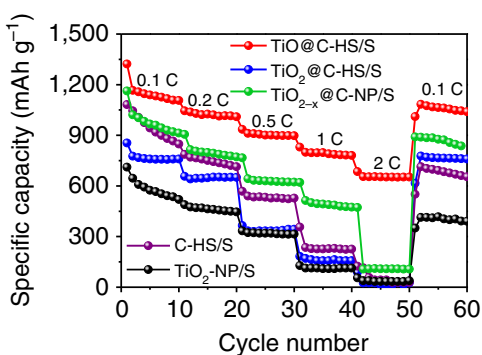

b

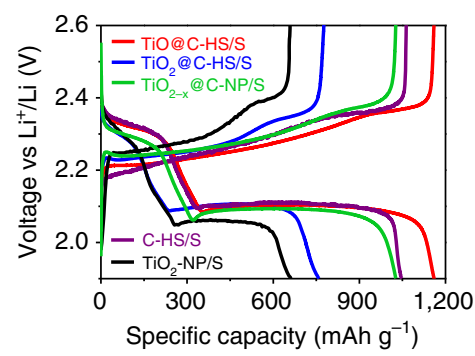

e

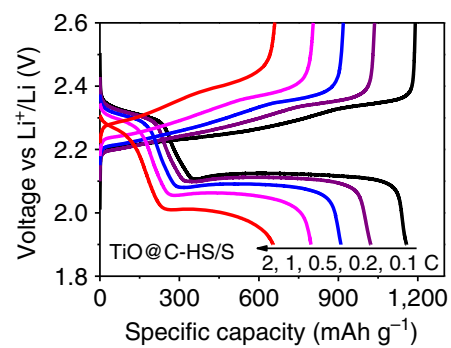

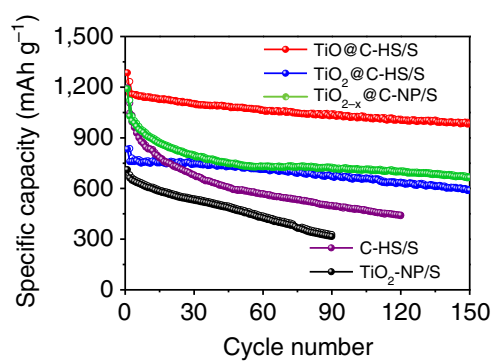

f

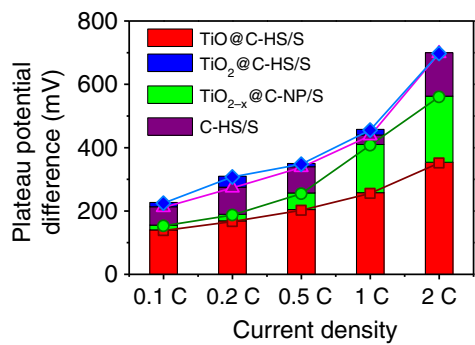

g

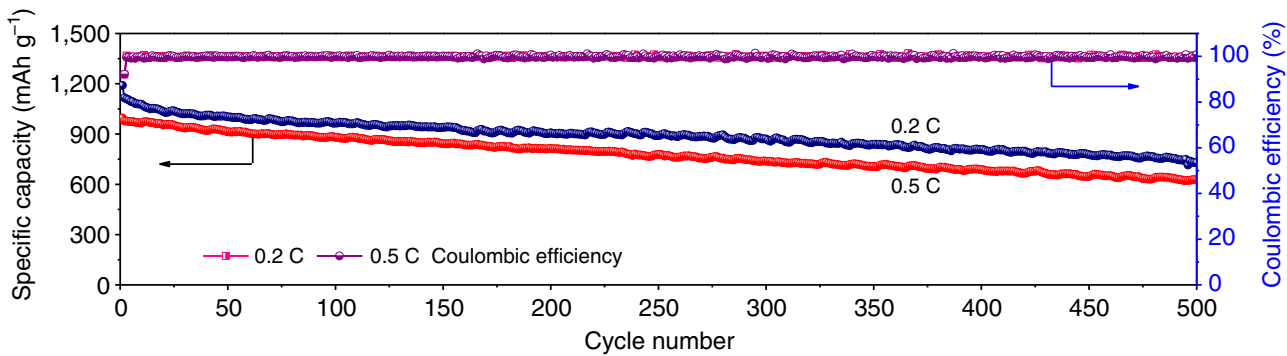

h

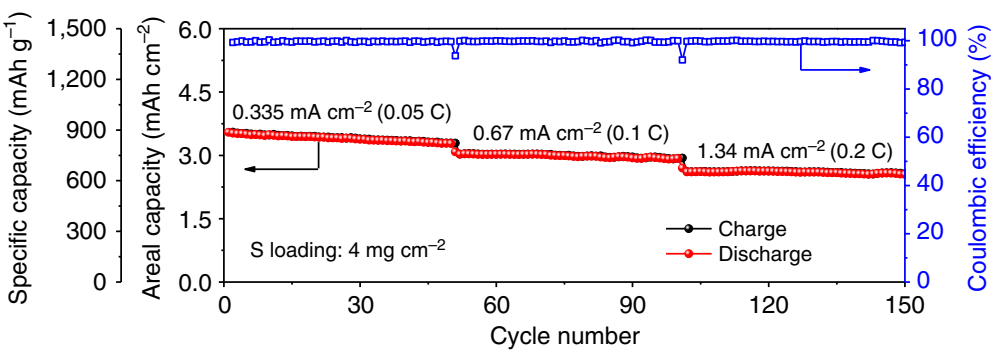

i

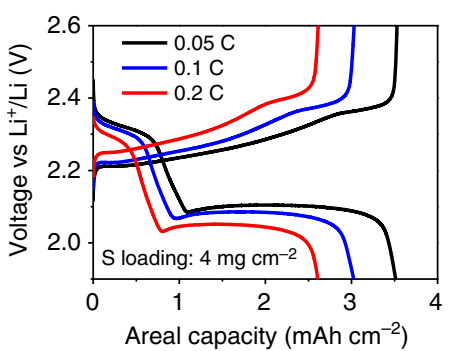

Figure 5 | Electrochemical evaluation of TiO@C-HS/S. (a) Nyquist plots before cycling from $1 \mathrm{MHz}$ to $100 \mathrm{mHz}$, (b) the second-cycle galvanostatic charge/discharge voltage profiles at $0.1 \mathrm{C},(\mathbf{c})$ cycle performances at $0.1 \mathrm{C},(\mathbf{d})$ rate capabilities and (f) the potential differences between the charge and discharge plateaus at various current densities of the TiO@C-HS/S,TiO $2 @ C-H S / S, T i O_{2-x} @ C-N P / S, C-H S / S$ and $\mathrm{TiO}_{2}-\mathrm{NP} / \mathrm{S}$ electrodes. (e) Voltage profiles at various current densities from 0.1 to $2 \mathrm{C}$ and $(\mathbf{g})$ prolonged cycle life and Coulombic efficiency at 0.2 and $0.5 \mathrm{C}$ of the TiO@C-HS/S electrode. (h) Areal capacities and (i) voltage profiles at various current densities from $0.335(0.05 \mathrm{C})$ to $1.34 \mathrm{~mA} \mathrm{~cm}^{-2}(0.2 \mathrm{C})$ of the TiO@C-HS/S electrode with high sulfur mass loading of $4.0 \mathrm{mg} \mathrm{cm}^{-2}$. 
samples, TiO@C-HS/S shows both highest first-discharge plateau at $\sim 2.33 \mathrm{~V}$ (corresponding to the reduction of sulfur to long-chain LiPSs) and longest second-discharge plateau at $\sim 2.1 \mathrm{~V}$ (the formation of short-chain LiPSs), suggesting much better redox reaction kinetics and more efficient utilization of the active sulfur material in TiO@C-HS/S. The cycling performance of five electrodes are compared at a relatively low current density of $0.1 \mathrm{C}$ (Fig. 5c). Benefitting from the high conductivity of $\mathrm{TiO}, \mathrm{Ti}_{4} \mathrm{O}_{7}$ and carbon, the electrodes of TiO@C-HS/S, TiO ${ }_{-x} @ \mathrm{C}-\mathrm{NP} / \mathrm{S}$ and C-HS/S deliver high initial discharge capacities of $1,285,1,190$ and $1,195 \mathrm{mAhg}^{-1}$, respectively, while $\mathrm{TiO}_{2} @ \mathrm{C}-\mathrm{HS} / \mathrm{S}$ and $\mathrm{TiO}_{2}-\mathrm{NP} / \mathrm{S}$ only give 833 and $711 \mathrm{mAh}^{-1}$, respectively. It can be indicated that higher conductivity of the hosts promises better reaction kinetics of the active sulfur material. However, after 150 cycles, the discharge capacities of $\mathrm{TiO}_{2-x} @ \mathrm{C}-\mathrm{NP} / \mathrm{S}$ drops to $664 \mathrm{mAhg}^{-1}$, corresponding to capacity retention of only 56\%. Another nanoparticle formed cathode of $\mathrm{TiO}_{2}-\mathrm{NP} / \mathrm{S}$ delivers even worse cycling stability with the capacity retention of only $44 \%$ after 90 cycles. Without polar material in the host, C-HS/S shows the fastest fading ratio among all groups, and retains a capacity of only $442 \mathrm{mAh} \mathrm{g}^{-1}$ after 120 cycles, which is $37 \%$ of the initial capacity. In contrast, the $\mathrm{TiO} @ \mathrm{C}-\mathrm{HS} / \mathrm{S}$ and $\mathrm{TiO}_{2} @ \mathrm{C}-\mathrm{HS} / \mathrm{S}$ electrodes show much better cycling stability with capacity retention of $76 \%$ and $71 \%$, respectively, suggesting that hollow structured polar hosts can effectively block the diffusion of polysulfides and enhance the cycling stability.

Next, the rate capabilities and electrode kinetics of these cathode materials are evaluated at various current densities (Fig. 5d). When the current density is increased successively from $0.1 \mathrm{C}$ to $0.2,0.5,1$ and $2 \mathrm{C}$, the TiO@C-HS/S electrode delivers high stabilized specific capacities of 1,146, 1,029, 910, 800 and $655 \mathrm{mAh} \mathrm{g}^{-1}$, respectively (Fig. 5d,e). When the current density is reduced back to $0.1 \mathrm{C}$, the discharge capacity of TiO@C-HS/S is recovered to $1,083 \mathrm{mAh}^{-1}$, indicating good stability of the cathode structure after the high rate discharging and charging test. Compared with TiO@C-HS/S, both of the $\mathrm{TiO}_{2-x} @ \mathrm{C}-\mathrm{NP} / \mathrm{S}$ and C-HS/S electrodes deliver obvious lower discharge capacities at various current densities from $0.1 \mathrm{C}$ to $2 \mathrm{C}$ (Fig. 5d, Supplementary Fig. 7a,b), which may be caused by the loss of active materials through LiPSs dissolution at the early cycles of the test. Although the $\mathrm{TiO}_{2} @ \mathrm{C}-\mathrm{HS} / \mathrm{S}$ electrode also shows good cycling stability, it shows an abrupt capacity drop when the current density is increased to $0.5 \mathrm{C}$, and has almost no capacity at $2 \mathrm{C}$ (Fig. 5d, Supplementary Fig. 7c). Because of the poor conductivity and ineffective LiPSs confinement of the bare $\mathrm{TiO}_{2}$ nanoparticles, the $\mathrm{TiO}_{2}-\mathrm{NP} / \mathrm{S}$ electrode shows the worst rate capability among all groups (Fig. $5 \mathrm{~d}$ ). The calculated potential differences between the charge/discharge voltage plateaus at various current densities further confirm that the TiO@C-HS/S electrode possesses much less polarization and better reaction kinetics than the other samples (Fig. 5f), which can be attributed to its excellent conductivity and efficiency for LiPSs adsorption. The prolonged cycle life of the TiO@C-HS/S electrode is tested at 0.2 and $0.5 \mathrm{C}$ for 500 cycles (Fig. $5 \mathrm{~g}$ ). After the initial discharge capacities of 1,190 and $1,066 \mathrm{mAhg}^{-1}$, the TiO@C-HS/S electrode delivers capacities of $750 \mathrm{mAhg}^{-1}$ at $0.2 \mathrm{C}$ and $630 \mathrm{mAhg}^{-1}$ at $0.5 \mathrm{C}$, respectively, corresponding to a small average capacity decay rate of $\sim 0.08 \%$ per cycle. The Coulombic efficiencies of the TiO@C-HS/S cells are >99\% during the cycling process (Fig. 5g).

Since high mass loading of active materials and high areal capacities are essential for the energy density of Li-S batteries, a thick TiO@C-HS/S electrode with areal sulfur loading of $4.0 \mathrm{mg} \mathrm{cm}^{-2}$ is further evaluated (Fig. 5h,i). Upon cycling at $0.05 \mathrm{C}$, a discharge capacity of $886 \mathrm{mAhg}^{-1}$ is delivered, corresponding to an areal capacity of $3.5 \mathrm{mAh} \mathrm{cm}^{-2}$. When the current density is increased to 0.1 and $0.2 \mathrm{C}$, the capacities are stabilized at above $730 \mathrm{mAh} \mathrm{g}^{-1}\left(2.9 \mathrm{mAh} \mathrm{cm}^{-2}\right)$ and $630 \mathrm{mAh} \mathrm{g}^{-1}\left(2.5 \mathrm{mAh} \mathrm{cm}^{-2}\right)$ over 50 cycles, respectively. The results of three different cells tested in the same condition demonstrate that the cells have good consistency, and the electrochemical performances are highly reproducible (Supplementary Fig. 8). The good cycling performance of a high mass-loading sulfur electrode requires good conductivity of the cathode materials, as well as efficient confinement of LiPSs. All these results show that TiO@C-HS is an attractive host material for the sulfur cathode of $\mathrm{Li}-\mathrm{S}$ batteries to achieve stable cycling and high energy density. By comparing with many similar cathodes based on metal oxides/sulfides hosts (Supplementary Table 1 and Supplementary Fig. 9), it can be noted that the TiO@C-HS/S electrode of this work exhibits much enhanced areal capacities and very attractive cycling stability at high sulfur loading. Although the areal capacity reported here is not the highest compared with some recently reported carbon-based electrodes ${ }^{51-53}$, we believe that by further optimizing the design for the $\mathrm{TiO} / \mathrm{C}$-based cathode and other components in the cell, it is very possible to significantly enhance the electrochemical performance of Li-S battery.

\section{Discussion}

The schematic illustrations of $\mathrm{Li}-\mathrm{S}$ batteries show the different effects of various nanostructured sulfur hosts on electrochemical performance (Fig. 6a,e,i,m,q). The TiO@C-HS is the only structure that possesses both advantages of high conductivity and effective LiPSs confinement. To further demonstrate the superiority of TiO@C-HS for stable cycling performance, the electrode films and separators are examined after cycling. Cells are disassembled at fully charged status after cycling tests, and all of the electrode films and separators are directly used for the characterization without any treatment. SEM observations show that in the fresh electrodes, TiO@C-HS/S and $\mathrm{TiO}_{2} @ \mathrm{C}-\mathrm{HS} / \mathrm{S}$ well maintain the spherical morphologies with unbroken shells (Fig. 6b,f), indicating good mechanical robustness of the hollow hosts for sustaining the electrode fabricating process. After cycling, the spherical TiO@C-HS/S and $\mathrm{TiO}_{2} @ \mathrm{C}-\mathrm{HS} / \mathrm{S}$ composites show almost no variation compared with the pristine status (Fig. $6 \mathrm{c}, \mathrm{g}$ ), and the electrode films are also in good intact forms as the fresh ones (insets of Fig. 6b,c,f,g). As for $\mathrm{TiO}_{2-x} @ \mathrm{C}-\mathrm{NP} / \mathrm{S}, \mathrm{TiO}_{2}-\mathrm{NP} / \mathrm{S}$ and $\mathrm{C}-\mathrm{HS} / \mathrm{S}$, compared with the fresh electrodes (Fig. 6j,n,r), the expanded layers of LiPSs are all clearly evident on the surfaces of the cycled electrodes (Fig. 6k,o,s), and some cracks are generated on the electrode films (Inset of Fig. 6k,o,s). Since the separator is closely compressed on the surface of cathode electrode in the tightly sealed coin cell, the area of orange LiPSs on separator could also reflect their dissolution from the cathode. The trace of dissolved LiPSs on the separator from the TiO@C-HS/S cell shows inconspicuous colour and the smallest area, implying the most effective restriction of LiPSs in the TiO@C-HS/S cathode (Fig. 6d). In contrast, the orange areas on the separators of the $\mathrm{TiO}_{2-x} @ \mathrm{C}-\mathrm{NP} / \mathrm{S}, \mathrm{TiO}_{2}-\mathrm{NP} / \mathrm{S}$ and $\mathrm{C}-\mathrm{HS} / \mathrm{S}$ cells are much larger, and the colours are more distinct than that of the TiO@C-HS/S and $\mathrm{TiO}_{2} @ \mathrm{C}-\mathrm{HS} / \mathrm{S}$ cells (Fig.6d,h,l,p,t), indicating that significant amount of LiPSs are dissolved from the $\mathrm{TiO}_{2-x} @ \mathrm{C}-\mathrm{NP} / \mathrm{S}$, $\mathrm{TiO}_{2}-\mathrm{NP} / \mathrm{S}$ and $\mathrm{C}-\mathrm{HS} / \mathrm{S}$ cathodes during the cycling. These observations are in good agreement with the electrochemical test results, and visually demonstrate that TiO@C-HS serves as a great sulfur host for Li-S batteries.

To better reveal the superiority of TiO@C-HS on restricting the diffusion of LiPSs, the bonding properties of $\mathrm{S}_{x}$ and $\mathrm{Li}_{2} \mathrm{~S}_{x}(x=1$, 


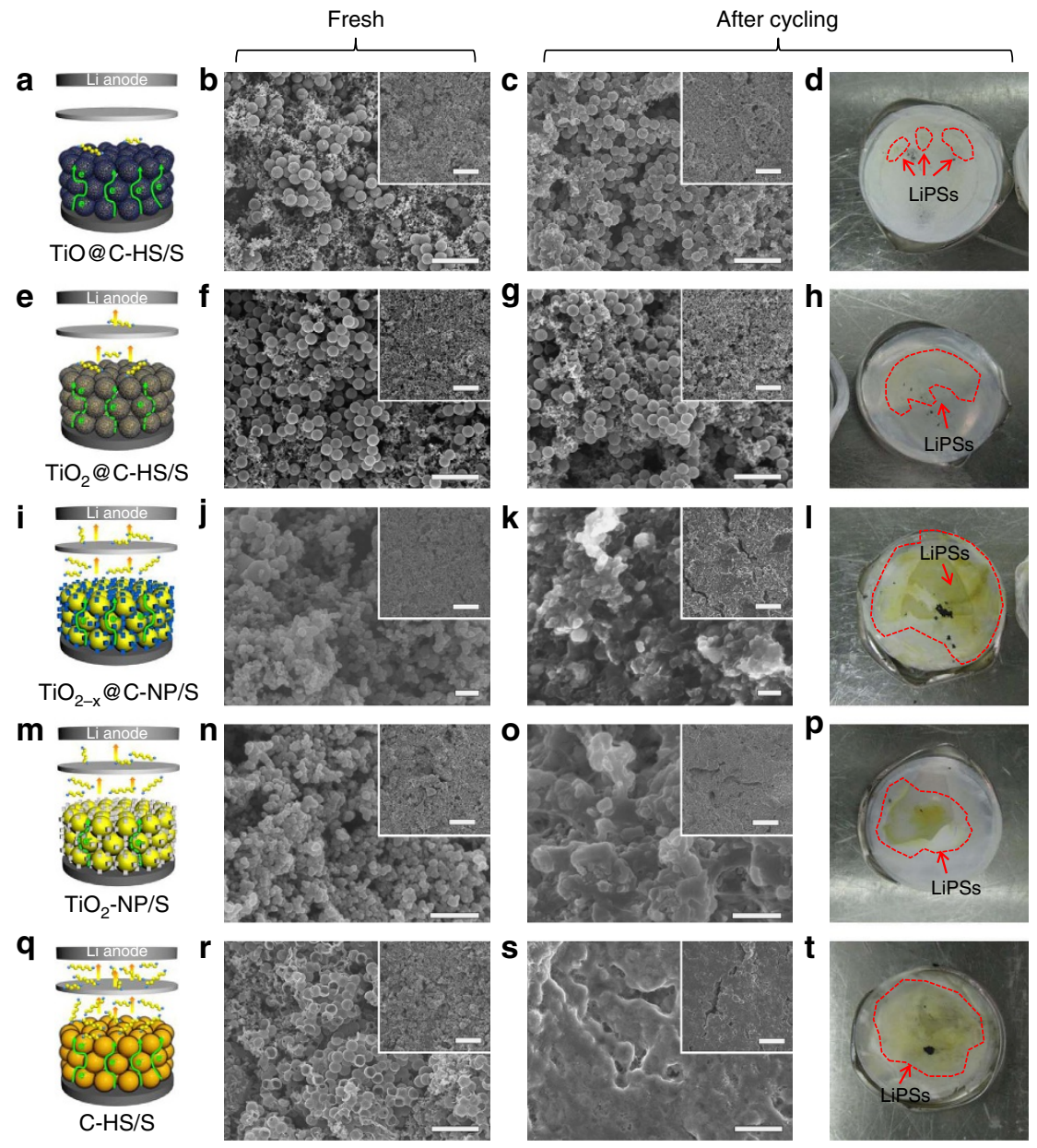

Figure 6 | Characterization of electrode films and separators during cycling. (a,e,i,m,q) Schematic illustration of the mechanisms during redox reaction, SEM images of $(\mathbf{b}, \mathbf{f}, \mathbf{j}, \mathbf{n}, \mathbf{r})$ fresh electrode films, $(\mathbf{c}, \mathbf{g}, \mathbf{k}, \mathbf{0}, \mathbf{s})$ cycled electrode films and $(\mathbf{d}, \mathbf{h}, \mathbf{l}, \mathbf{p}, \mathbf{t})$ digital photos of cycled separators of (a-d) TiO@C-HS/S,

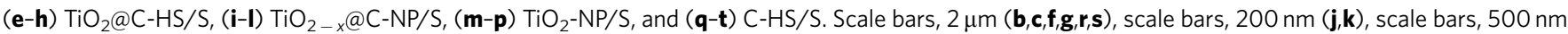
$(\mathbf{n}, \mathbf{o})$, scale bars, $10 \mu \mathrm{m}$ (insets of $(\mathbf{b}, \mathbf{c}, \mathbf{f}, \mathbf{g}, \mathbf{j}, \mathbf{k}, \mathbf{n}, \mathbf{o}, \mathbf{r}, \mathbf{s})$ ).

2 and 4) on $\mathrm{TiO}(001)$ (ref. 54) and rutile $\mathrm{TiO}_{2}$ (110) (ref. 55) are studied by DFT calculations. The adsorption energy $\left(E_{\mathrm{a}}\right)$ is calculated using the equation, $E_{\mathrm{a}}=E_{\mathrm{SS}}+E_{X}\left(X=\mathrm{S}, \mathrm{S}_{2}, \mathrm{~S}_{4}, \mathrm{Li}_{2} \mathrm{~S}\right.$, $\mathrm{Li}_{2} \mathrm{~S}_{2}$ and $\left.\mathrm{Li}_{2} \mathrm{~S}_{4}\right)-\mathrm{E}_{\text {total }}$. Here, $E_{\text {total }}, E_{\mathrm{SS}}$ and $E_{X}$ are the total energy of the whole system, substrate and molecular clusters, respectively, and the more negative $E_{\mathrm{a}}$ indicates the stronger adsorption capability. The calculated $E_{\mathrm{a}}$ of different adsorption systems are listed in Supplementary Table 2 and the optimized adsorption structures are shown in Supplementary Fig. 10. It is found that the $\mathrm{S}_{x} / \mathrm{Li}_{2} \mathrm{~S}_{x}$ adsorptions on $\mathrm{TiO}$ (001) are much stronger than on $\mathrm{TiO}_{2}$ (110) (Fig. 7a,b and Supplementary Table 2). More importantly, the adsorption energies of $S_{2}$ $(-2.711 \mathrm{eV})$ and $\mathrm{S}_{4}(-2.571 \mathrm{eV})$ on $\mathrm{TiO}(001)$ are about $1 \mathrm{eV}$ larger than the corresponding ones on $\mathrm{TiO}_{2}(110)(-1.914 \mathrm{eV}$ for $\mathrm{S}_{2}$ and $-1.134 \mathrm{eV}$ for $\mathrm{S}_{4}$ ), thus $\mathrm{TiO}(001)$ plane has a striking ability to adsorb $S_{x}$ clusters. The results indicate that, as the sulfur host, $\mathrm{TiO}$ can inhibit the dissolution of polysulfides clusters more effectively than rutile $\mathrm{TiO}_{2}$. To understand the underlying reason for the relative larger adsorption energies of both $\mathrm{S}_{x}$ and $\mathrm{Li}_{2} \mathrm{~S}_{x}$ compounds on TiO (001), the electronic properties of the $S_{x}$ and $\mathrm{Li}_{2} \mathrm{~S}_{x}$ adsorbed on $\mathrm{TiO}(001)$ and $\mathrm{TiO}_{2}$ (110) systems are further analysed. The charge-differences show that the $\mathrm{S}$ atoms prefer to interact with the two surface oxygen atoms with the typical covalent bonds in $\mathrm{S}_{x} / \mathrm{TiO}_{2}$ (110) structures (Fig. 7c). This phenomenon could also be supported by other XPS and theoretical calculation results reported elsewhere ${ }^{42}$. Notably, with the extension of $S$ chains $\left(S \rightarrow S_{2} \rightarrow S_{4}\right)$, the chemical interaction between $S_{x}$ and $\mathrm{TiO}_{2}(110)$ becomes weaker. In contrast, when $S_{x}$ clusters adsorb on $\mathrm{TiO}(001)$, the $\mathrm{S}$ atoms strongly interact with the surface $\mathrm{Ti}_{5 \mathrm{c}}$ atoms with the character of the ionic interaction (Fig. 7e), which also leads to the more pronounced elongation of the S-S bonds in sulfur chains on $\mathrm{TiO}(001)$ than that on $\mathrm{TiO}_{2}$ (110) (Supplementary Table 2). These should be the main reason for the relatively larger adsorption energies of $\mathrm{S}_{x}$ on $\mathrm{TiO}(001)$. On the other hand, the strong interaction between $\mathrm{Li}_{2} \mathrm{~S}_{x}$ and $\mathrm{TiO}$ (001) mainly originates from the large portion of the low coordinated $\mathrm{Ti}_{5 \mathrm{c}}$ active sites on $\mathrm{TiO}(001)$, since all $\mathrm{Ti}$ on the $\mathrm{TiO}$ (001) surface are $\mathrm{Ti}_{5 c}$, while the ratio of $\mathrm{Ti}_{5 c}$ and $\mathrm{Ti}_{6 c}$ is $1: 1$ on the $\mathrm{TiO}_{2}$ (110) surface. Thus, the TiO host can provide stronger chemical adsorption energies for $\mathrm{Li}_{2} \mathrm{~S}_{\mathrm{x}}$ due to the formation of both $\mathrm{Li}-\mathrm{O}$ and $\mathrm{Ti}-\mathrm{S}$ bonds. Based on these DFT calculation results, it is reasonable to assume that the electrochemical performance of the TiO@C-HS/S composite originates from the unique surface chemical properties of $\mathrm{TiO}$.

In summary, we have designed a sulfur host based on highly conductive polarTiO@C hollow nanospheres for lithium-sulfur batteries. This host can maximize the effectiveness of moderating LiPSs diffusion and enhance the redox reaction kinetics of sulfur species at the same time. Benefiting from the excellent conductivity and strong LiPSs adsorption capability of TiO@C shells, the TiO@C/S composite cathode delivers high discharge capacities of $>1,100 \mathrm{mAhg}^{-1}$ at $0.1 \mathrm{C}$, and exhibits stable cycle 
a

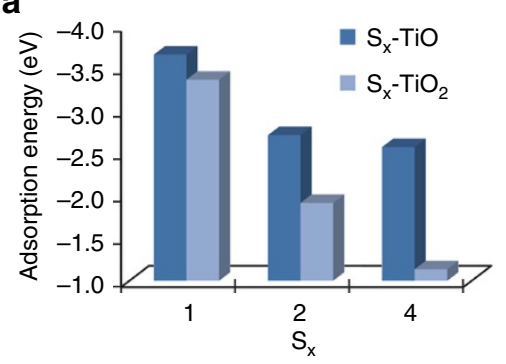

C

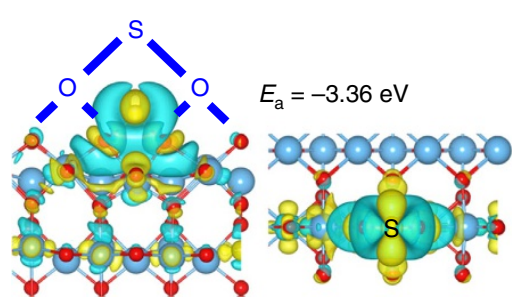

e

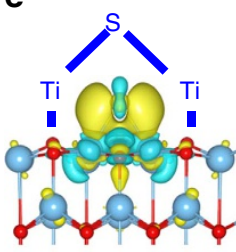

Side view

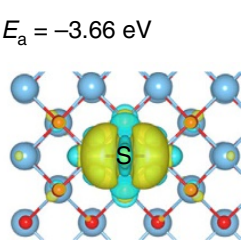

Top view

b

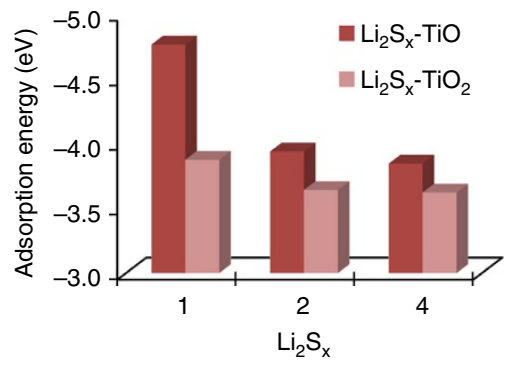

d

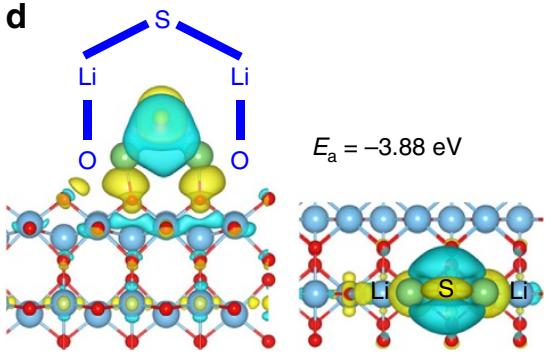

$\mathbf{f}$

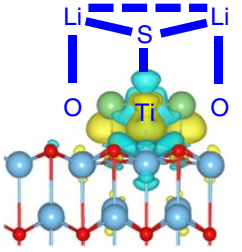

Side view

\section{$E_{\mathrm{a}}=-4.77 \mathrm{eV}$}

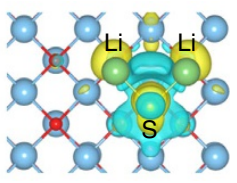

Top view

Figure 7 | DFT analysis of adsorption energies of $\mathbf{S}$ species on TiO (001) and $\mathbf{T i O}_{\mathbf{2}}$ (110). Adsorption energies for (a) $\mathrm{S}_{x}$ and $(\mathbf{b}) \mathrm{Li}_{2} \mathrm{~S}_{x}(x=1,2$ and 4$)$ compounds on $\mathrm{TiO}(001)$ and $\mathrm{TiO}_{2}$ (110) surfaces. Isosurface of the charge density difference for (c) $\mathrm{S}$ and $(\mathbf{d}) \mathrm{Li}_{2} \mathrm{~S}$ adsorbed on $\mathrm{TiO}_{2}(110)$, and (e) $\mathrm{S}$ and (f) $\mathrm{Li}_{2} \mathrm{~S}$ adsorbed on $\mathrm{TiO}$ (001). Yellow surfaces correspond to charge gains and blue surfaces correspond to an equivalent charge lost. To make the plot clear, the isovalues are defined as 0.002 in all cases.

life up to 500 cycles at 0.2 and $0.5 \mathrm{C}$ with a small capacity decay rate of $0.08 \%$ per cycle. In addition, when the areal loading of sulfur is increased to $4.0 \mathrm{mg} \mathrm{cm}^{-2}$, the TiO@C/S electrode can provide high areal capacities at various current densities with good stability and high Coulombic efficiency. This work overcomes the major limitations associated with other polar and nonpolar sulfur hosts, and may open up the prospect of constructing more efficient nanostructures for moderating the diffusion of LiPSs and enhancing the reaction kinetics of sulfur. Only with such high-efficiency sulfur cathodes, high-energy lithium-sulfur batteries can become possible in future.

\section{Methods}

Preparation of TiO@C-HS and other control host materials. PS nanospheres were prepared by a previously reported method ${ }^{48}$. $\mathrm{TiO}_{2}$ was firstly coated on PS via a method reported by our group ${ }^{49}$. Typically, $\sim 110 \mathrm{mg}$ of PS was dispersed in $35 \mathrm{ml}$ of ethanol, followed by adding $0.32 \mathrm{~g}$ of hexadecylamine and $0.8 \mathrm{ml}$ of ammonium hydroxide. After stirring for $10 \mathrm{~min}, 0.2 \mathrm{ml}$ of titanium isopropoxide was dropped into the dispersion under vigorous stirring. After reaction for $1 \mathrm{~h}$, PS@TiO 2 was collected by centrifugation and washed several times with ethanol and deionized water, and then dispersed in $150 \mathrm{ml}$ of Tris-buffer aqueous solution $\left(10 \mathrm{mmol}^{-1}\right)$ under ultrasonication. After that, $80 \mathrm{mg}$ of dopamine was added into the dispersion with magnetic stirring overnight.PS@TiO $@ P D A$ was collected by centrifugation and washed several times with deionized water and ethanol, dried at $70^{\circ} \mathrm{C}$ for $12 \mathrm{~h}$. Finally, TiO@C-HS was prepared by annealing PS@TiO ${ }_{2} @ P D A$ in a reductive atmosphere of $\mathrm{N}_{2} / \mathrm{H}_{2}(95: 5)$ mixture gas at $1,000^{\circ} \mathrm{C}$ for $4 \mathrm{~h}$ with a heating rate of $5^{\circ} \mathrm{C} \mathrm{min}^{-1}$. $\mathrm{TiO}_{2} @ \mathrm{C}$ - $\mathrm{HS}$ was obtained by annealing the same precursor of $\mathrm{PS} @ \mathrm{TiO}_{2} @ \mathrm{PDA}$ at $900^{\circ} \mathrm{C}$ for $2 \mathrm{~h}$ in the same atmosphere. For the synthesis of $\mathrm{TiO}_{2-x} @ \mathrm{C}-\mathrm{NP}, 100 \mathrm{mg}$ of commercial $\mathrm{TiO}_{2}$ nanoparticles

(Aeroxide P25, ACROS Organics) and $80 \mathrm{mg}$ of dopamine were dispersed in

$150 \mathrm{ml}$ of Tris-buffer aqueous solution $\left(10 \mathrm{mmoll}^{-1}\right)$ under ultrasonication, and then stirred for $12 \mathrm{~h}$. The resultant $\mathrm{TiO}_{2} / \mathrm{PDA}$ composite was washed several times with deionized water and ethanol by centrifugation, and dried at $70^{\circ} \mathrm{C}$ for $12 \mathrm{~h}$. $\mathrm{TiO}_{2-x} @ \mathrm{C}-\mathrm{NP}$ was obtained by annealing $\mathrm{TiO}_{2} / \mathrm{PDA}$ at $1,000^{\circ} \mathrm{C}$ for $4 \mathrm{~h}$ in the same atmosphere of $\mathrm{N}_{2} / \mathrm{H}_{2}$ (95:5). To prepare C-HS, $80 \mathrm{mg}$ of PS and $160 \mathrm{mg}$ of dopamine were dispersed into $150 \mathrm{ml}$ of Tris-buffer solution $\left(10 \mathrm{mmoll}^{-1}\right)$ with magnetic stirring overnight. The resultant PS@PDA was washed with water and ethanol for several times, and then dried at $70^{\circ} \mathrm{C}$ for $12 \mathrm{~h}$. The C-HS sample was obtained by annealing PS@PDA at $900^{\circ} \mathrm{C}$ for $2 \mathrm{~h}$ in $\mathrm{N}_{2}$ atmosphere. $\mathrm{TiO}_{2}-\mathrm{NP}$ (Aeroxide P25) was heated at $110^{\circ} \mathrm{C}$ for $12 \mathrm{~h}$ in oven before use.

Preparation of TiO@C-HS/S and other control composites. The mixture of TiO@C-HS and sulfur powder (1:5, weight ratio) was sealed in a glass vessel under argon protection, and heated at $300^{\circ} \mathrm{C}$ for $4 \mathrm{~h}$ in a quartz tube furnace for composing sulfur with TiO@C-HS. Then, the composite was placed in an open porcelain boat, and heated at $200{ }^{\circ} \mathrm{C}$ for $2 \mathrm{~h}$ in the quartz tube furnace under flowing argon gas to evaporate the extra sulfur that exists outside the TiO@C-HSs. After cooling down, TiO@C-HS/S was obtained. The preparation process of $\mathrm{TiO}_{2} @ \mathrm{C}$ $\mathrm{HS} / \mathrm{S}$ is the same with the above mentioned method. Since the sulfur contents in both $\mathrm{TiO} @ \mathrm{C}-\mathrm{HS} / \mathrm{S}$ and $\mathrm{TiO}_{2} @ \mathrm{C}-\mathrm{HS} / \mathrm{S}$ are around $70 \mathrm{wt} \%$, the sulfur contents in $\mathrm{TiO}_{2-x} @ \mathrm{C}-\mathrm{NP} / \mathrm{S}, \mathrm{C}-\mathrm{HS} / \mathrm{S}$ and $\mathrm{TiO}_{2}-\mathrm{NP} / \mathrm{S}$ are controlled to be about the same for a fair comparison. The $\mathrm{TiO}_{2-x} @ \mathrm{C}-\mathrm{NP} / \mathrm{S}$ composite was prepared by heating the mixture of $\mathrm{TiO}_{2-x} @ \mathrm{C}-\mathrm{NP}$ and sulfur $(3: 7, \mathrm{w} / \mathrm{w})$ at $155^{\circ} \mathrm{C}$ for $12 \mathrm{~h}$ in a sealed glass vessel under argon protection. The C-HS/S and $\mathrm{TiO}_{2}-\mathrm{NP} / \mathrm{S}$ composites were prepared by the same melt-diffusion method with $\mathrm{TiO}_{2-x} @ \mathrm{C}-\mathrm{NP} / \mathrm{S}$.

Structural and phase characterization. The morphologies and structures of the samples were characterized by field-emission scanning electron microscope (JEOL-6700), TEM (JEOL, JEM-2010). Linear elemental scanning was recorded using EDX spectroscopy attached to TEM (JEOL, JEM-2100F). The crystal phases of the products were determined by a Bruker D2 Phaser X-Ray Diffractometer with $\mathrm{Cu} \mathrm{K} \alpha$ radiation $(\lambda=1.5406 \AA)$. Sulfur or carbon contents of samples were determined by thermogravimetric analysis (Shimadzu DRG-60) in nitrogen or air flow, respectively. 
Electrochemical measurements. The electrode film was prepared by mixing $80 \mathrm{wt} \%$ of active material, $10 \mathrm{wt} \%$ of conductive carbon and $10 \mathrm{wt} \%$ of polyvinylidene fluoride in N-methylpyrrolidone, then the slurry was casted on the aluminium foil and dried at $70{ }^{\circ} \mathrm{C}$ overnight. The areal mass loading of sulfur is about $1.5 \mathrm{mg} \mathrm{cm}^{-2}$. The thick electrode film with sulfur loading of $4.0 \mathrm{mg} \mathrm{cm}^{-2}$ was composed of active material, conductive carbon and binder in the weight ratio of 70:20:10. The 2032-type coin cells were assembled using Celgard 2300 membrane as separator and $\mathrm{Li}$ metal as anode. The electrolyte was prepared by dissolving $1.0 \mathrm{moll}^{-1}$ lithium bis(trifluoromethanesulfonyl) imide in a mixture of 1,3-dioxolane and dimethoxymethane $(1: 1, \mathrm{v} / \mathrm{v})$ with addition of $0.2 \mathrm{moll}^{-1}$ of $\mathrm{LiNO}_{3}$. The volume of electrolyte injected in coin cells is controlled as about $20 \mu \mathrm{l}$ per $1 \mathrm{mg}$ of electrode. The galvanostatic charge/discharge measurements were performed in a voltage range of $1.9-2.6 \mathrm{~V}$ using a NEWARE battery tester. The capacities were calculated based on the mass of sulfur. The electrochemical impedance measurements were carried out at $5 \mathrm{mV}$ ac oscillation amplitude over the frequency range of $100 \mathrm{kHz}$ to $100 \mathrm{MHz}$.

Computational method. The calculations were performed using the Vienna Ab Initio Simulation Package in the framework of DFT ${ }^{56-58}$. The projector augmented wave pseudopotential was adopted and the generalized gradient approximation with the Perdew-Burke-Ernzerhof exchange-correlation (PBE) functional was used to treat the exchange-correlation interaction between electron $s^{59}$. The cutoff energy of the projector augmented plane-wave basis set is $500 \mathrm{eV}$ to ensure an accuracy of the energy of $1 \mathrm{meV}$ per atom. The full geometry optimizations are carried out with the convergence thresholds of $10^{-5} \mathrm{eV}$ and $1 \times 10^{-2} \mathrm{eV} \AA^{-1}$ for total energy and ionic force, respectively. To get a reasonable description of the adsorption system, all the substrates are modelled with the periodic supercell. The $k$-space integration uses the Monkhorst-Pack scheme on a $2 \times 2 \times 1$ and $1 \times 2 \times 2$ mesh for TiO $(001)$ and $\mathrm{TiO}_{2}$ (110) substrates, respectively. A $4 \times 4$ supercell slab of TiO containing 160 atoms $\left(\mathrm{Ti}_{80} \mathrm{O}_{80}\right)$ was used. As for $\mathrm{TiO}_{2}(110)$, we employed a $2 \times 4$ supercell containing 192 atoms $\left(\mathrm{Ti}_{64} \mathrm{O}_{128}\right)$ as the substrate.

Data availability. The authors declare that the data supporting the findings of this study are available within the article and its Supplementary Information Files. All other relevant data supporting the findings of this study are available on request.

\section{References}

1. Bruce, P. G., Freunberger, S. A., Hardwick, L. J. \& Tarascon, J. M. Li- $\mathrm{O}_{2}$ and Li-S batteries with high energy storage. Nat. Mater. 11, 19-29 (2012).

2. Manthiram, A., Fu, Y., Chung, S. H., Zu, C. \& Su, Y. S. Rechargeable lithiumsulfur batteries. Chem. Rev. 114, 11751-11787 (2014).

3. Chung, W. J. et al. The use of elemental sulfur as an alternative feedstock for polymeric materials. Nat. Chem. 5, 518-524 (2013).

4. Wei, S., Ma, L., Hendrickson, K. E., Tu, Z. \& Archer, L. A. Metal-sulfur battery cathodes based on PAN-sulfur composites. J. Am. Chem. Soc. 137, 1214312152 (2015).

5. Zhou, W., Yu, Y., Chen, H., DiSalvo, F. J. \& Abruna, H. D. Yolk-shell structure of polyaniline-coated sulfur for lithium-sulfur batteries. J. Am. Chem. Soc. 135, 16736-16743 (2013)

6. Li, Z., Huang, Y., Yuan, L., Hao, Z. \& Huang, Y. Status and prospects in sulfur-carbon composites as cathode materials for rechargeable lithium-sulfur batteries. Carbon 92, 41-63 (2015).

7. Liang, J., Sun, Z.-H., Li, F. \& Cheng, H.-M. Carbon materials for Li-S batteries: Functional evolution and performance improvement. Energy Storage Mater. 2, 76-106 (2016)

8. Qie, L. \& Manthiram, A. A facile layer-by-layer approach for high-arealcapacity sulfur cathodes. Adv. Mater. 27, 1694-1700 (2015).

9. Su, Y. S. \& Manthiram, A. Lithium-sulphur batteries with a microporous carbon paper as a bifunctional interlayer. Nat. Commun. 3, 1166 (2012).

10. Zhou, G. et al. A graphene-pure-sulfur sandwich structure for ultrafast, long-life lithium-sulfur batteries. Adv. Mater. 26, 625-631 (2014).

11. Huang, J. Q. et al. Ionic shield for polysulfides towards highly-stable lithium-sulfur batteries. Energy Environ. Sci. 7, 347-353 (2014).

12. Suo, L., Hu, Y. S., Li, H., Armand, M. \& Chen, L. A new class of solvent-in-salt electrolyte for high-energy rechargeable metallic lithium batteries. Nat. Commun. 4, 1481 (2013).

13. Lin, Z., Liu, Z., Fu, W., Dudney, N. J. \& Liang, C. Lithium polysulfidophosphates: a family of lithium-conducting sulfur-rich compounds for lithium-sulfur batteries. Angew. Chem. Int. Ed. 52, 7460-7463 (2013).

14. Cuisinier, M. et al. Unique behaviour of nonsolvents for polysulphides in lithium-sulphur batteries. Energy Environ. Sci. 7, 2697-2705 (2014).

15. Seh, Z. W. et al. Stable cycling of lithium sulfide cathodes through strong affinity with a bifunctional binder. Chem. Sci. 4, 3673 (2013).

16. Wang, L., Dong, Z., Wang, D., Zhang, F. \& Jin, J. Covalent bond glued sulfur nanosheet-based cathode integration for long-cycle-life Li-S batteries. Nano Lett. 13, 6244-6250 (2013).
17. Li, G. et al. Acacia senegal-inspired bifunctional binder for longevity of lithium-sulfur batteries. Adv. Energy Mater. 5, 1500878 (2015).

18. Fang, X. \& Peng, H. A revolution in electrodes: recent progress in rechargeable lithium-sulfur batteries. Small 11, 1488-1511 (2015).

19. Ji, X. L., Lee, K. T. \& Nazar, L. F. A highly ordered nanostructured carbon-sulphur cathode for lithium-sulphur batteries. Nat. Mater. 8, 500-506 (2009).

20. Li, Z. et al. A highly ordered meso@microporous carbon-supported sulfur@smaller sulfur core-shell structured cathode for Li-S batteries. ACS Nano 8, 9295-9303 (2014).

21. Xin, S. et al. Smaller sulfur molecules promise better lithium-sulfur batteries. J. Am. Chem. Soc. 134, 18510-18513 (2012).

22. Zhao, M. Q. et al. Unstacked double-layer templated graphene for high-rate lithium-sulphur batteries. Nat. Commun. 5, 3410 (2014).

23. Pang, Q., Liang, X., Kwok, C. Y. \& Nazar, L. F. Review-the importance of chemical interactions between sulfur host materials and lithium polysulfides for advanced lithium-sulfur batteries. J. Electrochem. Soc. 162, A2567-A2576 (2015).

24. Peng, H. J. \& Zhang, Q. Designing host materials for sulfur cathodes: from physical confinement to surface chemistry. Angew. Chem. Int. Ed. 54, 11018-11020 (2015).

25. Pang, Q. et al. A nitrogen and sulfur dual-doped carbon derived from polyrhodanine@cellulose for advanced lithium-sulfur batteries. Adv. Mater. 27, 6021-6028 (2015).

26. Zhou, G., Paek, E., Hwang, G. S. \& Manthiram, A. Long-life Li/polysulphide batteries with high sulphur loading enabled by lightweight three-dimensional nitrogen/sulphur-codoped graphene sponge. Nat. Commun. 6, 7760 (2015).

27. Yang, C. P. et al. Insight into the effect of boron doping on sulfur/carbon cathode in lithium-sulfur batteries. ACS Appl. Mater. Interfaces 6, 8789-8795 (2014).

28. Wang, Z. et al. Enhancing lithium-sulphur battery performance by strongly binding the discharge products on amino-functionalized reduced graphene oxide. Nat. Commun. 5, 5002 (2014).

29. Ma, L. et al. Enhanced Li-S batteries using amine-functionalized carbon nanotubes in the cathode. ACS Nano 10, 1050-1059 (2015).

30. Li, Z., Zhang, J. T., Chen, Y. M., Li, J. \& Lou, X. W. Pie-like electrode design for high-energy density lithium-sulfur batteries. Nat. Commun. 6, 8850 (2015).

31. Zhou, J. W. et al. Rational design of a metal-organic framework host for sulfur storage in fast, long-cycle Li-S batteries. Energy Environ. Sci. 7, 2715-2724 (2014).

32. Liang, X., Garsuch, A. \& Nazar, L. F. Sulfur cathodes based on conductive MXene nanosheets for high-performance lithium-sulfur batteries. Angew. Chem. Int. Ed. 54, 3907-3911 (2015).

33. Jiang, J. et al. Encapsulation of sulfur with thin-layered nickel-based hydroxides for long-cyclic lithium-sulfur cells. Nat. Commun. 6, 8622 (2015).

34. Zhang, J., Hu, H., Li, Z. \& Lou, X. W. Double-shelled nanocages with cobalt hydroxide inner shell and layered double hydroxides outer shell as highefficiency polysulfide mediator for lithium-sulfur batteries. Angew. Chem. Int. Ed. 55, 3982-3986 (2016).

35. Ji, X., Evers, S., Black, R. \& Nazar, L. F. Stabilizing lithium-sulphur cathodes using polysulphide reservoirs. Nat. Commun. 2, 325 (2011).

36. Seh, Z. W. et al. Sulphur- $\mathrm{TiO}_{2}$ yolk-shell nanoarchitecture with internal void space for long-cycle lithium-sulphur batteries. Nat. Commun. 4, 1331 (2013).

37. Yao, H. et al. Improving lithium-sulphur batteries through spatial control of sulphur species deposition on a hybrid electrode surface. Nat. Commun. 5, 3943 (2014).

38. Liang, X. et al. A highly efficient polysulfide mediator for lithium-sulfur batteries. Nat. Commun. 6, 5682 (2015).

39. Li, Z., Zhang, J. \& Lou, X. W. Hollow carbon nanofibers filled with $\mathrm{MnO}_{2}$ nanosheets as efficient sulfur hosts for lithium-sulfur batteries. Angew. Chem. Int. Ed. 54, 12886-12890 (2015).

40. Seh, Z. W. et al. Two-dimensional layered transition metal disulphides for effective encapsulation of high-capacity lithium sulphide cathodes. Nat. Commun. 5, 5017 (2014).

41. Yuan, Z. et al. Powering lithium-sulfur battery performance by propelling polysulfide redox at sulfiphilic hosts. Nano Lett. 16, 519-527 (2016).

42. Pang, Q., Kundu, D., Cuisinier, M. \& Nazar, L. F. Surface-enhanced redox chemistry of polysulphides on a metallic and polar host for lithium-sulphur batteries. Nat. Commun. 5, 4759 (2014).

43. Tao, X. et al. Strong sulfur binding with conducting magneli-phase $\mathrm{Ti}_{\mathrm{n}} \mathrm{O}_{2 \mathrm{n}-1}$ nanomaterials for improving lithium-sulfur batteries. Nano Lett. 14, 5288-5294 (2014).

44. Pang, Q., Kundu, D. \& Nazar, L. F. A graphene-like metallic cathode host for long-life and high-loading lithium-sulfur batteries. Mater. Horiz. 3, 130-136 (2016). 
45. Clarke, R. L. \& Harnsberger, S. K. New electrically conductive ceramic - a fundamental advance in electrode technology. Am. Lab. 20, 8-10 (1988).

46. Smith, J. R., Walsh, F. C. \& Clarke, R. L. Electrodes based on magnéli phase titanium oxides: the properties and applications of ebonex materials. J. Appl. Electrochem. 28, 1021-1033 (1998).

47. Gusev, A. A. \& Avvakumov, E. G. Conducting materials based on nanodispersed titanium monoxide. Sci. Sinter. 39, 295-299 (2007).

48. Zhang, J., Chen, Z., Wang, Z., Zhang, W. \& Ming, N. Preparation of monodisperse polystyrene spheres in aqueous alcohol system. Mater. Lett. 57, 4466-4470 (2003).

49. Guan, B., Yu, l., Li, J. \& Lou, X. W. A universal cooperative assembly-directed method for coating of mesoporous $\mathrm{TiO}_{2}$ nanoshells with enhanced lithium storage properties. Sci. Adv. 2, e1501554 (2016).

50. Jayaprakash, N., Shen, J., Moganty, S. S., Corona, A. \& Archer, L. A. Porous hollow carbon@sulfur composites for high-power lithium-sulfur batteries. Angew. Chem. Int. Ed. 50, 5904-5908 (2011).

51. Song, J. X. et al. Nitrogen- doped mesoporous carbon promoted chemical adsorption of sulfur and fabrication of high- areal- capacity sulfur cathode with exceptional cycling stability for lithium- sulfur batteries. Adv. Funct. Mater. 24, 1243-1250 (2014).

52. Xu, T. et al. Mesoporous carbon-carbon nanotube-sulfur composite microspheres for high-areal-capacity lithium-sulfur battery cathodes. ACS Appl. Mater. Interfaces 5, 11355-11362 (2013).

53. Fang, R. et al. 3D interconnected electrode materials with ultrahigh areal sulfur loading for Li-S batteries. Adv. Mater. 28, 3374-3382 (2016).

54. Pabon, B. M. et al. Formation of titanium monoxide (001) single-crystalline thin film induced by ion bombardment of titanium dioxide (110). Nat. Commun. 6, 6147 (2015).

55. Henderson, M. A. \& Lyubinetsky, I. Molecular-level insights into photocatalysis from scanning probe microscopy studies on $\mathrm{TiO}_{2}(110)$. Chem. Rev. 113, 4428-4455 (2013)

56. Blöchl, P. E. Projector augmented-wave method. Phys. Rev. B 50, 17953-17979 (1994).

57. Kresse, G. \& Furthmüller, J. Efficient iterative schemes forab initiototal-energy calculations using a plane-wave basis set. Phys. Rev. B 54, 11169-11186 (1996).

58. Kresse, G. \& Joubert, D. From ultrasoft pseudopotentials to the projector augmented-wave method. Phys. Rev. B 59, 1758-1775 (1999).
59. Perdew, J. P., Burke, K. \& Ernzerhof, M. Generalized gradient approximation made simple. Phys. Rev. Lett. 77, 3865-3868 (1996).

\section{Acknowledgements}

X.W.L. is grateful to the Ministry of Education (Singapore) for financial support through the AcRF Tier 1 funding (Grant RG12/14; M4011258).

\section{Author contributions}

Z.L. and X.W.L. conceived the idea and co-wrote the manuscript. Z.L. and J.Z. carried out the materials synthesis and the electrochemical evaluation. L.-M.L. and D.W. carried out the DFT calculation. J.Z. and B.G. helped materials characterizations. All the authors discussed the results and commented on the manuscript.

\section{Additional information}

Supplementary Information accompanies this paper at http://www.nature.com/ naturecommunications

Competing financial interests: The authors declare no competing financial interests.

Reprints and permission information is available online at http://npg.nature.com/ reprintsandpermissions/

How to cite this article: Li, Z. et al. A sulfur host based on titanium monoxide@carbon hollow spheres for advanced lithium-sulfur batteries. Nat. Commun. 7, 13065 doi: 10.1038/ncomms13065 (2016).

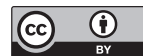

This work is licensed under a Creative Commons Attribution 4.0 International License. The images or other third party material in this article are included in the article's Creative Commons license, unless indicated otherwise in the credit line; if the material is not included under the Creative Commons license, users will need to obtain permission from the license holder to reproduce the material. To view a copy of this license, visit http://creativecommons.org/licenses/by/4.0/

(C) The Author(s) 2016 